\title{
WIRKUNG CARCINOSTATISCHER ÄTHYLENIMIN-VERBINDUNGEN AUF DEN DPN-GEHALT THERAPIERESISTENER TUMOREN*
}

\author{
P. Scriba, H. Kröger und H. Holzer \\ Physiologisch-Chemisches Institut der Universität \\ Freiburg im Breisgau
}

(Received 5 December 1960)

\begin{abstract}
Preliminary experiments with the ascites form of the relatively therapeuticresistant DS-tumour demonstrated that the decrease in DPN content and the inhibition of glycolysis in the neoplastic cells under the infuence of ethylenimine compounds were delayed, in comparison with the rapid changes observed in Ehrlich-ascites carcinomas. Extending these observations, the therapeutic effect of carcinostatic ethylenimine compounds was studied in rats bearing solid forms of either the Jensen sarcoma or the therapeutic-resistant DS-tumour. During the course of the experiments the DPN content of the tumours was followed. In the Jensen sarcoma the DPN content decreased sharply as early as the first day following administration of the carcinostatic, while the DS-tumour, in contrast, showed no clear change during the first six days. A cure was obtained in nine out of thirteen cases of the Jensen-sarcoma rats, while no cure was observed in six DS-tumour-bearing rats. These experiments further support our hypothesis, that carcinostatic ethylenimine compounds are therapeutically effective though they depress the DPN content in the tumour.
\end{abstract}

CARCINOSTATISCHE Äthylenimin-Verbindungen hemmen in vitro die anaerobe und aerobe Glykolyse von Ascitestumorzellen ${ }^{3,4}$ durch Senkung des Gehaltes an DPN. ${ }^{3,5,8}$ Auf Grund der von Warburg ${ }^{7}$ gefundenen engen Beziehungen zwischen aerober Glykolyse und malignem Wachstum war zu vermuten, dass die Glykolysehemmung auch am Mechanismus der Heilung gewisser Tumoren im lebenden Tier entscheidend beteiligt sei. Hierfür sprechen die folgenden Beobachtungen:

(1) Nach intravenöser Verabreichung heilender Dosen von Äthyleniminverbindungen an Jensen-Sarkom tragende Ratten findet man ein weitgehend auf den Tumor beschränktes, d.h. gewebsspezifisches Absinken des DPN-Gehaltes, das dem makroskopisch sichtbaren Einschmelzen des Tumors vorausgeht. ${ }^{8}$

(2) Wird gleichzeitig mit heilenden Dosen von Äthylenimin-Verbindungen an Jensen-Sarkom-Ratten Nikotinsäureamid verabreicht, so bleibt das Absinken des DPN aus, und völlig parallel dazu wird die Heilung verhindert. ${ }^{1,8}$

* Uber die Ergebnisse der vorliegenden Arbeit wurde auszugsweise von P. Scriba beim IV Internationalen Biochemiekongress in Wien am 2 Sept. $1958^{1}$ und am 27.1.1959 vor der Medizinischen Gesellschaft Freiburg im Breisgau ${ }^{2}$ vorgetragen.

+ Abkürzungen: DPN $=$ Diphosphopyridinnucleotid; NSA $=$ Nikotinsäureamid; TCE $=$ Trichloressigsäure; DS-Tumor siehe Methoden, E 39 und A 139 siehe Präparate. 
(3) Beim Vergleich der zur Heilung von Tumoren und zur Senkung des DPN-Gehaltes notwendigen Dosen verschiedener carcinostatischer ÄthyleniminVerbindungen findet man, dass auch hier Senkung des DPN-Gehaltes und Heilwirkung weitgehend parallel gehen."

Zur weiteren Prüfung der Frage, wie weitgehend Heilwirkung und Wirkung auf den DPN-Gehalt von Tumoren sich entsprechen, wurde in der vorliegenden Arbeit die Wirkung von Äthylenimin-Verbindungen auf zwei verschiedene Tumoren, und zwar auf einen relativ therapieresistenten und einen der Therapie zugänglichen Tumor verglichen. Wir fanden in gutem Einklang mit den vorstehend aufgeführten früheren Versuchen wiederum Parallelität zwischen heilender und DPN senkender Wirkung.

\section{METHODEN}

\section{Tumoren}

Die Versuche wurden zum Teil an einem Ehrlich-Ascites-Carcinom durchgeführt, das uns von Herrn Professor Warburg freundlicherweise überlassen wurde und dessen Eigenschaften früher beschrieben wurden. ${ }^{6}, 10,11$ Herrn Professor Druckrey danken wir für das Jensen-Sarkom und für ein Carcino-Sarkom, das durch Dimethylaminostilbenfütterung (DS-Tumor) bei Ratten hervorgerufen wurde. ${ }^{12}$ Der DSTumor wurde als solid, subkutan wachsender Tumor und als Ascitestumor untersucht. Es wurden Wistar-Ratten verwandt. Die Transplantation und die Technik der E 39 Behandlung wurden früher beschrieben. ${ }^{8}$

\section{Manometrie}

Es wurde die übliche Technik der Manometrie mit der Warburg-Apparatur angewandt (cf. Holzer et al. ${ }^{4}$ ). Jeweils $30-50 \mathrm{~mm}^{3}$ Zellen pro $\mathrm{ml}$ Krebs-Ringer-Bicarbonatlösung wurden bei $37^{\circ} \mathrm{C}$ im Warburg-Gefäss inkubiert. Das pH wurde durch $\mathrm{CO}_{2}$ Begasung auf 6,0 eingestellt und Glucose in $0,025 \mathrm{M}$ Endkonzentration zugesetzt.

\section{DPN-Bestimmung}

Die Aufarbeitung der Zellsuspensionen und der soliden Tumoren sowie die DPNBestimmung mit dem optischen Test nach Warburg wurden früher beschrieben. ${ }^{6,}, 8,10,11$

\section{PRÄPARATE}

Von der Firma Bayer-Leverkusen wurden uns freundlicherweise die Substanzen E 39 (2,5-Di-n-propoxy-3,6-bisäthyleniminobenzochinon-1,4) und A 139 (2,5-Dimethoxy-äthoxy-3,6-bisäthylenimino-benzochinon-1,4) zur Verfügung gestellt. Wir möchten auch an dieser Stelle den Herren Professor Dr. G. Domagk und Dr. S. Petersen für die Überlassung der Präparate danken.

\section{ERGEBNISSE}

\section{Versuche mit Ascites-Tumoren}

In Tabelle 1 sind Ergebnisse von manometrischen Glykolysemessungen und von DPN-Bestimmungen in Zellen der Ascitesform des von Druckrey et al ${ }^{12}$ als besonders therapieresistent beschriebenen DS-Tumors zusammengestellt. Aus den in der untersten Zeile angegebenen Glykolyse-Daten sieht man, dass die hier untersuchten Zellen sich im Prinzip genau so verhalten, wie die früher von uns untersuchten Zellen des 
Ehrlichschen Ascites-Carcinoms:6, 10 Zusatz der carcinostatischen ÄthyleniminVerbindung A 139 bewirkt eine kräftige Hemmung der Glykolyse, gleichzeitiger Zusatz von Nikotinsäureamid hebt, wie die letzte Kolonne der Tabelle zeigt, die Glykolysehemmung auf. Betrachtet man jedoch die Ergebnisse der DPN-Analysen, so sieht man, dass der Abfall des DPN-Gehaltes sehr spät einsetzt. Nach 80 min Inkubation mit A 139 ist der DPN-Gehalt erst um ca. $40 \%$ gesunken.

\section{TABelle 1. Wirkung von NSA auf anaerobe Glykolyse und DPN-Gehalt von ASCITESZELLEN DES DS-TUMORS BEI INKUBATION MIT UND OHNE A 139}

Manometrie und DPN-Bestimmung sind unter Methoden beschrieben. Pro Ansatz (Gesamtvolumen $6 \mathrm{ml}$ ) wurden $162 \mathrm{~mm}^{3}$ Zellen eingesetzt. Die Konzentration des A 139 betrug $0,18 \times 10^{-3} \mathrm{M}$ $(=6 \mathrm{mg} \%$, die des NSA $0,016 \mathrm{M}$.

\begin{tabular}{|c|c|c|c|c|}
\hline & \multicolumn{4}{|c|}{ Behandlung } \\
\hline & Kontrolle & A139 & NSA & A139 NSA \\
\hline $\begin{array}{l}\underset{\text { Mole DPN }}{\mu \text { mm }^{3} \text { Zellen }} \times 10^{4} \\
\text { nach } 80 \mathrm{~min} \text { Inkubation* }\end{array}$ & 2,73 & 1,67 & 3,18 & 2,38 \\
\hline $\begin{array}{l}\frac{\mu \text { Mole DPN }}{\text { mm }^{3} \text { Zellen }} \times 10^{4} \\
\text { nach } 240 \text { min Inkubation* }\end{array}$ & 1,75 & $<0,2$ & 2,45 & 1,67 \\
\hline $\begin{array}{l}\mathrm{mm}^{3} \mathrm{CO}_{2} \text { pro } \mathrm{mm}^{3} \text { Zellen } \\
\text { von der } 210-240 \mathrm{~min} \\
\text { nach Glucosezusatz }\end{array}$ & 0.52 & 0,11 & 0,51 & 0,45 \\
\hline
\end{tabular}

* Es handelt sich bei den beiden Versuchsreihen zur DPN-Bestimmung um verschiedene Versuche.

Es war zu vermuten, dass die durch den langsamen DPN-Abfall bewirkte sehr spät einsetzende Glykolysehemmung mit den besonderen Resistenzeigenschaften des hier untersuchten Ascites-DS-Tumors zusammenhängt. Deshalb wurde dieser mit dem Ehrlichschen Ascites-Carcinom verglichen. In Abb. 1 sind Glykolysewerte und der DPN-Gehalt der Zellen nach Abschluss der Glykolysemessungen (rechteckig eingerahmte Werte) eingetragen. Während im Ehrlichschen Ascites-Carcinom die Glykolyse rasch abfällt und nach etwa $60 \mathrm{~min}$ vollständig gehemmt ist, setzt eine Hemmung der Glykolyse beim DS-Tumor erst nach ca. $100 \mathrm{~min}$ ein. Vollständige Hemmung ist nach etwa 240 min erreicht. Zu diesem Zeitpunkt ist beim DS-Tumor ebenso wie beim Ehrlich-Ascites-Carcinom bei völliger Glykolysehemmung der DPN-Gehalt auf einen mit unserer Methode nicht mehr messbaren Wert abgesunken. Der besonders therapieresistente DS-Tumor zeichnet sich demnach auch durch Unempfindlichkeit gegen den DPN-senkenden und glykolysehemmenden Einfluss von Äthyleniminverbindungen aus.

\section{Versuche mit soliden Tumoren}

Um die Therapieresistenz, d.h. das Eintreten bzw. Ausbleiben von Heilerfolgen, in Zahlen angeben zu können, wurden die in Tabelle 2 niedergelegten Versuche mit den soliden Formen des DS-Tumors und des Jensen-Sarkoms durchgeführt. Der DS-Tumor war gegen die in diesen Versuchen eingesetzte Äthylenimin-Verbindung 
E 39 weitgehend resistent: es wurden keine Heilungen, sondern lediglich Lebensverlängerungen beobachtet. Beim Jensen-Sarkom wurden 9 von 13 Tieren geheilt (das Ausbleiben eines Rezidivs innerhalb einer fünfmonatigen Nachbeobachtung wurde von uns als Heilung bezeichnet). Die Ergebnisse von DPN-Bestimmungen in den Tumoren völlig parallel geführter Tiere sind in Abb. 2 eingetragen. Während

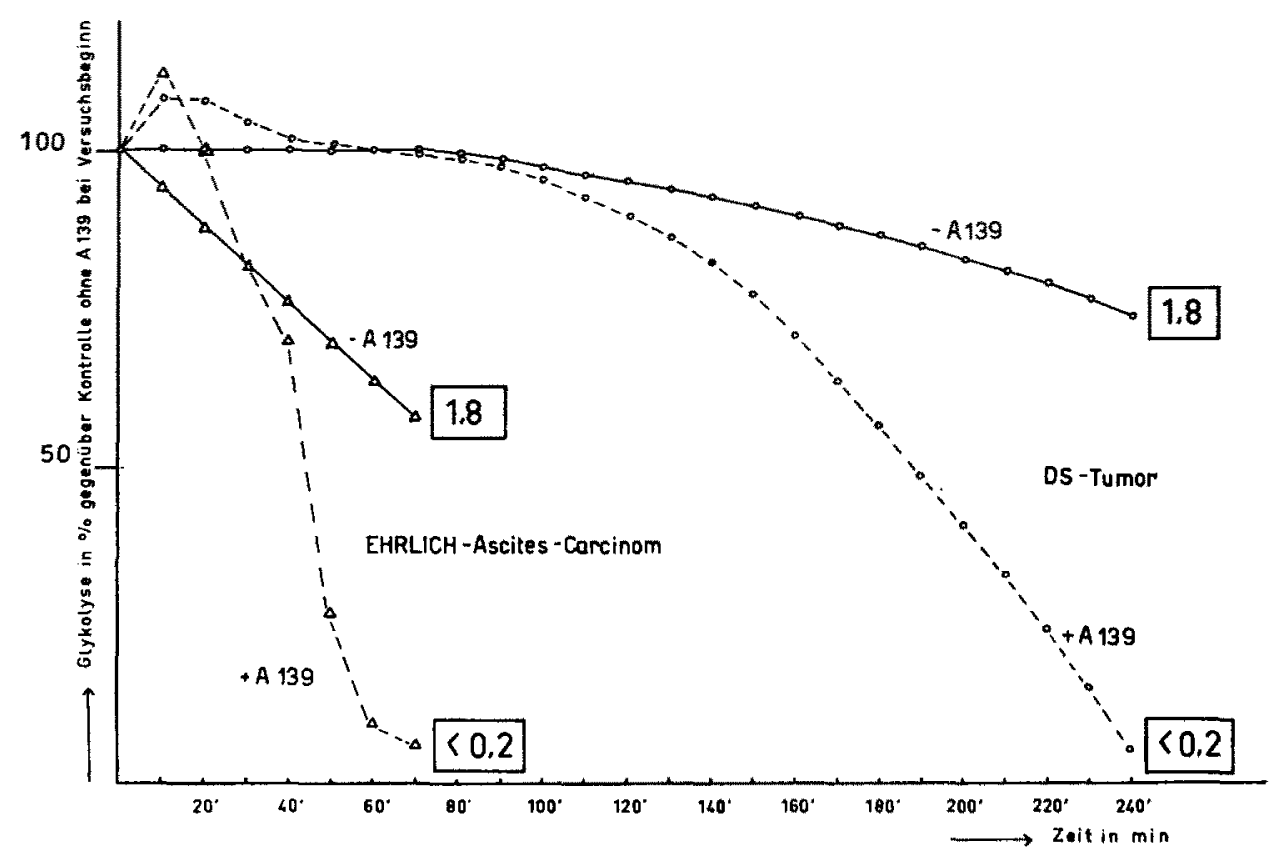

ABB. 1. Anaerobe Glykolyse und DPN-Gehalt von Ehrlich-Ascites-Carcinom und Ascitesform des DS-Tumors. Endkonzentration an A 139:0,18 $\times 10^{-3} \mathrm{M}$. Manometrie siehe Methoden. Nach der letzten in die Abbildung eingetragenen manometrischen Ablesung wurden die Zellen mit TCE abgetötet und aufgeschlossen. Die Ergebnisse der mit dem klarzentrifugierten Uberstand durchgeführten DPN-Bestimmungen in $\mu$ Mole DPN $\times 10^{4}$ pro $\mathrm{mm}^{3}$ Zellen sind rechteckig eingerahmt in die Abbildung eingetragen.

TABelle 2. Vergleich der Therapieempfindlichikett gegenüber E 39 von JensenSARKOM UND SOLIDEM DS-TUMOR BEI WISTAR-RATTEN

Die Technik der Beimpfung und die Behandlung der Tiere wurden früher beschrieben. ${ }^{8}$

\begin{tabular}{l|c|c|c}
\multicolumn{1}{c}{ Tumor } & $\begin{array}{c}\text { Zahl der mit 3,1 mg E 39 pro kg } \\
\text { behandelten Tiere }\end{array}$ & $\begin{array}{c}\text { Geheilt } \\
\text { (5 Monate Nachbeobachtung) }\end{array}$ & Gestorben \\
\hline Jensen-Sarkom & 13 & 9 & $4^{*}$ \\
\hline DS-Tumor & 6 & 0 & $6 *$ \\
\hline
\end{tabular}

* Die behandelten Tiere überlebten die unbehandelten Kontrolltiere um 8 Tage (Jensen-Sarkom) bzw. 27 Tage (DS-Tumor).

beim Jensen-Sarkom der von uns schon früher mitgeteilte ${ }^{8}$ rasche Abfall des DPNGehaltes eintritt, nimmt im DS-Tumor der DPN-Gehalt in den ersten Tagen nach Verabreichung von E 39 nicht ab. Ein Vergleich der in Tabelle 2 angegebenen Heilungserfolge mit dem in Abb. 2 niedergelegten Verhalten des DPN-Gehaltes 
demonstriert die Parallelität von Heilwirkung und Wirkung auf den DPN-Gehalt der Tumoren.

\section{ZUSAMMENFASSUNG}

In orientierenden Versuchen mit der Ascites-Form des relativ therapieresistenten DS-Tumors wurde gefunden, das der DPN-Abfall und die Glykolysehemmung durch Äthylenimin-Verbindungen im Vergleich zum Ehrlich-Ascites-Carcinom verzögert

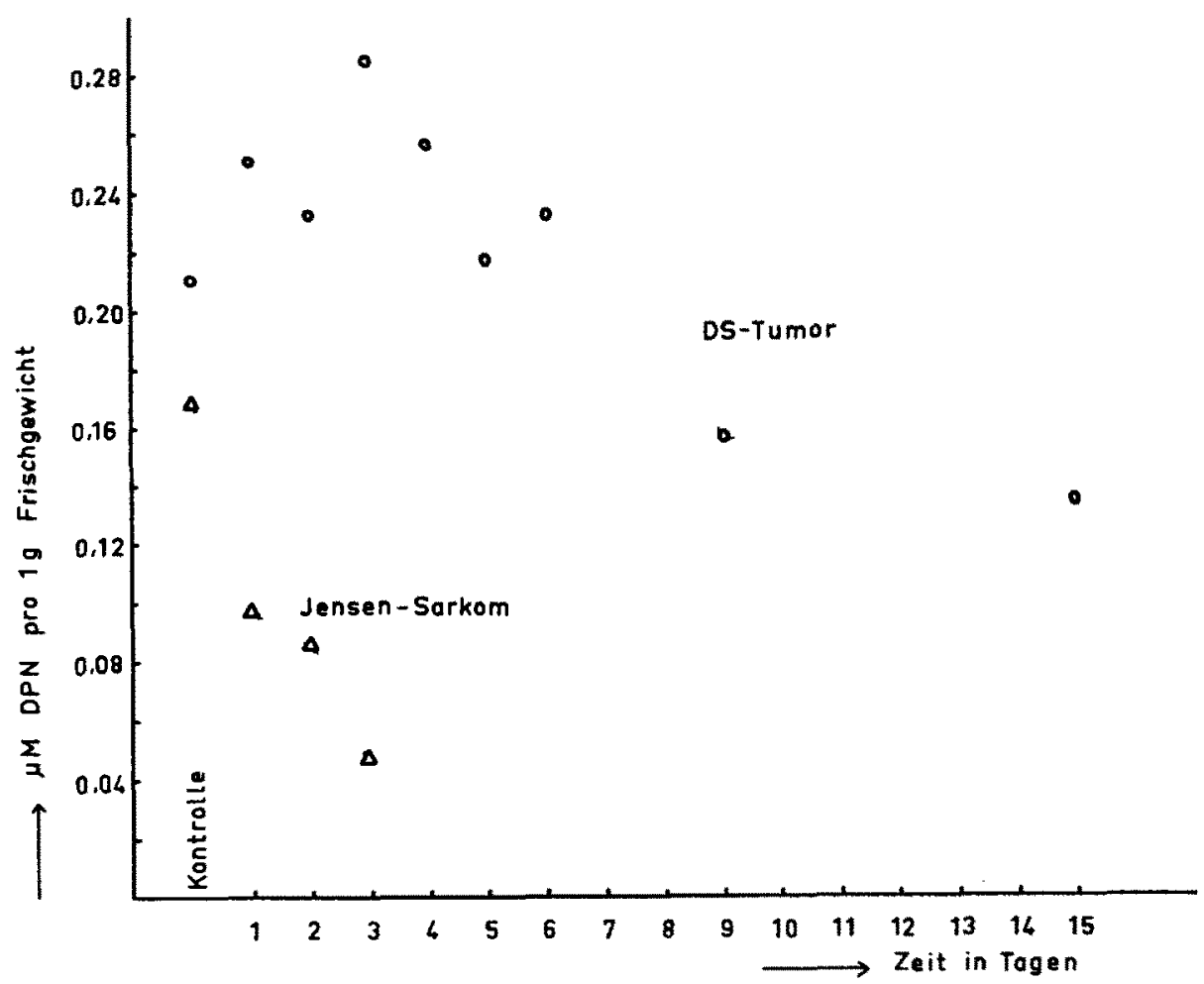

ABB. 2. DPN-Gehalt in Jensen-Sarkom und DS-Tumor (solide Form) nach einmaliger Verabreichung von 3,1 mg E 39/kg Körpergewicht. Die als "Kontrolle" (nach 0 Tagen) eingetragenen Werte wurden von Tieren gemessen, die nicht mit $\mathrm{E} 39$ behandelt wurden.

auftreten. Aufbauend auf diesen Befund wurde die Heilwirkung carcinostatischer Äthylenimin-Chinon-Verbindungen bei Jensen-Sarkom- bzw. den therapieresistenten DS-Tumor-tragenden Ratten studiert. Parallel dazu wurde der DPN-Gehalt in den Tumoren gemessen. Im Jensen-Sarkom fallt der DPN-Gehalt bereits einen Tag nach Verabreichung des Carcinostaticums stark ab, während im DS-Tumor in den ersten 6 Tagen keine deutliche Veränderung zu beobachten ist. Dementsprechend wurde bei den Jensen-Sarkom tragenden Tieren in 9 von 13 Fällen eine Heilung beobachtet, während bei keinem der 6 DS-Tumor tragenden Tiere Heilung eintrat. Die vorgelegten Versuche sind eine weitere Stütze für unsere Annahme, dass carcinostatische Äthylenimin-Verbindungen durch Senkung des DPN-Gehaltes in Tumoren deren Heilung bewirken. 
Anerkennungen-Wir danken der Deutschen Forschungsgemeinschaft und dem Bundesministerium für Atomkernenergie und Wasserwirtschaft für Sachbeihilfen.

\section{LITERATUR}

1. P. SCRIBA, H. KRÖGER und H. Holzer, IV Internationaler Biochemie Kongress, Wien, 1-6 September 1958, Sektion 14, 9. International Abstracts of Biological Sciences, 1958.

2. P. SCRIBA, Klin. Wschr. 37, 678 (1959).

3. I. M. RoITT, Biochem. J. 63, 300 (1956).

4. H. Holzer, G. Sedlmayr und A. Kemnitz, Biochem. Z. 328, 163 (1956).

5. H. Holzer und G. Sedlmayr, Ber. ges. Physiol. 189, 120 (1957).

6. H. Holzer, P. Glogner und G. Sedlmayr, Biochem. $Z$. 330, 59 (1958).

7. O. Warburg, Über den Stoffwechsel der Tumoren. Julius Springer, Berlin (1926).

8. H. Holzer und H. KröGER, Biochem. Z. 330, 579 (1958).

9. H. KröGer, B. Ulrich und H. Holzer, Arzneimittel-Forsch. 9, 598 (1959).

10. P. SCRIBA, S. SCHNeIDER und H. HOLzER, Z. Krebsforsch. 63, 547 (1960).

11. P. SCRJBA, Dissertation, Medizinische Fakultät der Universität Freiburg i. Br. (1959).

12. H. Druckrey, D. Schmähl, P. Danneberg, K. Kaiser, H. A. Nieper, H. W. Lo, R. Mecke JR, J. von EINEM und W. DischleR, Arzmeimittel-Forsch. 6, 539 (1956). 